\title{
New method for electric field and potential calculations in Hall plates
}

Johan Raman, Pieter Rombouts and Ludo Weyten

This document is an author's draft version submitted for publication Electronics Letters

The actual version was published as [1].

\section{REFERENCES}

[1] J. Raman, P. Rombouts, and L. Weyten, "Method for electric field and potential calculations in Hall plates," Electron. Lett., vol. 49, no. 1, pp. 33-34, Jan 32013. 


\section{New method for electric field and potential calculations in Hall plates}

\section{J. Raman, P. Rombouts and L. Weyten}

Electrostatic field problems occurring in Hall plates are difficult to solve, mainly because of a non-standard boundary condition defining an oblique angle of the electric field w.r.t. an isolating boundary. In this letter, a new approach for solving Hall-related field problems is presented. Compared to prior approaches, the technique leads more easily to closed-form expressions for the electric field, and allows to obtain voltage-related Hall characteristics into numerically well conditioned forms.

Introduction: While the Hall effect has been studied for a long time [1-4], calculating the field and potential within a Hall plate still proves to be challenging. The core of the problem is the unusual oblique angle boundary condition (BC) that applies at isolating boundaries, which is different from standard Dirichlet, Neumann, and mixed-type conditions [1,2].

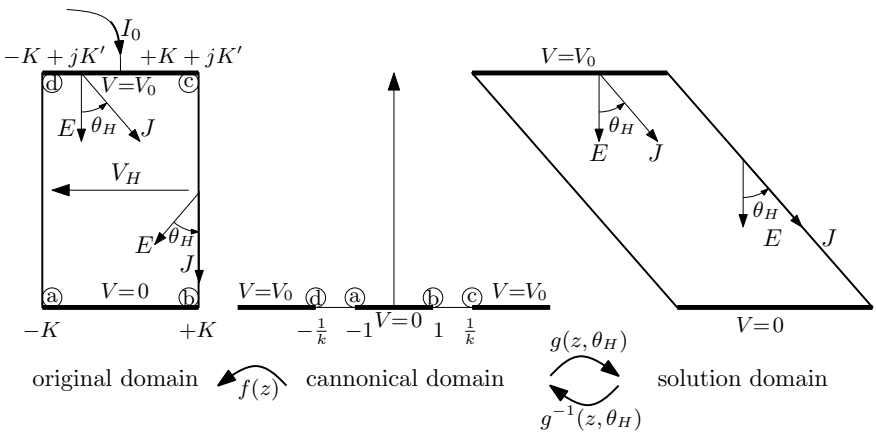

Fig. 1 Conventional approach to solving the Hall problem: find a conformal map from the original problem domain (left side) to a geometry for which the solution - in this case a uniform field - can be seen by inspection (right side).

An important prior method for solving the Hall problems goes back to the work of [4]. This method relies on the observation that for a particular shape of the problem domain, the problem has a trivial solution: a uniform electric field, and hence a linearly varying potential. For the problem of a rectangular Hall plate with two contacts, the situation is depicted in fig. 1 (right side). The problem then reduces to finding a conformal map between the artificial solution domain and the original domain (left side). This task is further decomposed into finding first a map $f(z)$ from a canonical domain (middle) onto the original domain, and a second one by inverting a mapping $g\left(z, \theta_{H}\right)$ from the canonical domain to the solution domain. A complicating factor here is that the shape of the solution domain depends on the Hall angle $\theta_{H}$ between $\mathbf{E}$ and $\mathbf{J}$. While for polygonal regions these two sub-mappings are of the Schwarz-Christoffel type, and hence are fairly easy to write down as complex integrals, finding a closed-form solution of the integral is even for simple geometries very complicated $[3,4]$. Therefore, in practice, numerical integration is required $[3,5]$. Since two mappings are involved, one being dependent on $\theta_{H}$, the prior solution method is computationally involved. The new method presented hereafter focuses on obtaining analytic solutions for the electric field, resorting only to numeric integration as the last step (to obtain the potential).

Equations and boundary conditions: We consider a thin sample in the xyplane, with a $2 \mathrm{D}$ electric field $\mathbf{E}(x, y)$ and current density $\mathbf{J}(x, y)$ within it. In the presence of a homogeneous magnetic field perpendicular to the sample $\left(\mathbf{B}=B_{0} \mathbf{e}_{z}\right)$, the constitutive relation becomes:

$$
\mathbf{E}(x, y)=\rho \mathbf{J}(x, y)+\mu_{H} B_{0} \rho\left(\mathbf{J}(x, y) \times \mathbf{e}_{z}\right)
$$

In this, $\rho$ denotes the conductance, $\mu_{H}$ the Hall mobility, and $\mathbf{e}_{z}$ is the unit vector in the z-direction. Using Maxwell's equations, it can be shown that both $\mathbf{E}$ and $\mathbf{J}$ can be derived from a potential $\left(\mathbf{E}=-\nabla V_{E}\right.$, $\mathbf{J}=-\nabla V_{J}$ ), with $V_{E}$ and $V_{J}$ solutions of Laplace's equation. It is well known that such $2 \mathrm{D}$ problems can also be described with complex-valued analytical functions. These are the complex potentials $w_{E}$ and $w_{J}$, which are requiring to be analytic in the problem domain. The latter implies that the associated potential $V_{E}=\operatorname{Re}\left[w_{E}\right]$ and $V_{J}=\operatorname{Re}\left[w_{J}\right]$ automatically solve Laplace's equation in this domain. The link between $w_{E}$ (resp. $w_{J}$ ) and the field components $E_{x}$ and $E_{y}$ (resp. $J_{x}$ and $J_{x}$ ) is

$$
\frac{d w_{E}}{d z}=-E_{x}+j E_{y} \stackrel{\text { def }}{=} E(z), \quad \frac{d w_{J}}{d z}=-J_{x}+j J_{y} \stackrel{\text { def }}{=} J(z)
$$

where we have introduced the function $E(z)$ (resp. $J(z)$ ) as a complex representation of the electric field (resp. the current density). The vector relation (1) can now be written in complex notation, and the result can be integrated using (2) to yield

$$
J(z)=\frac{\cos \theta_{H}}{\rho} e^{j \theta_{H}} \cdot E(z) \rightarrow w_{J}=\frac{\cos \theta_{H}}{\rho} e^{j \theta_{H}} \cdot w_{E}
$$

In this, $\theta_{H}=\arctan \left(\mu_{H} B_{0}\right)$ represents the Hall angle, and integration constants are, without loss of generality, set to zero. The above relations indicate that electric field and current density are related through a simple scale factor (which depends on the Hall angle).

The BCs of the Hall problem are as follows: at metallic contacts we have a prescribed voltage $V_{E}$, while at isolated parts of the boundary $\mathbf{J} \cdot \mathbf{n}=0$, with $\mathbf{n}$ the normal of the boundary curve. The prescribed voltage hinders the derivation of solutions for the fields. We deal with this by relaxing this $\mathrm{BC}$ : we require only that contacts have a constant voltage, but we do not enforce a particular value. The relaxed $\mathrm{BC}$ can be expressed as $\mathbf{E} \cdot \mathbf{t}=0$, with $\mathbf{t}$ denoting the tangent vector at the boundary point.

The BCs can be put in an equivalent complex form. For the tangent vector $\mathbf{t}=t_{x} \mathbf{e}_{x}+t_{y} \mathbf{e}_{x}$, we define $t=t_{x}+j t_{y}$ as the associated complex representation. Combined with (2), it can be derived that $\operatorname{Re}[t \cdot E(z)]=$ $-t_{x} E_{x}-t_{y} E_{y}=-\mathbf{E} \cdot \mathbf{t}$, which is zero at contact points. Let $\theta_{t}$ denote the angle of $t$ w.r.t. the $\mathrm{x}$-axis, then $t=e^{j \theta_{t}}$, and the $\mathrm{BC}$ at contacts becomes:

$$
\text { At } z \in \text { contact boundary: } \operatorname{Re}\left[e^{j \theta_{t}(z)} \cdot E(z)\right]=0
$$

Through the same procedure, the normal can be represented as $n=n_{x}+$ $j n_{y}=e^{j \theta_{n}}$, with $\theta_{n}$ the angle of the normal w.r.t. the $\mathrm{x}$-axis. At isolated boundary points, $\operatorname{Re}\left[e^{j \theta_{n}} \cdot J(z)\right]=0$, which in view of (3) becomes:

$$
\text { At } z \in \text { isolated boundary: } \operatorname{Re}\left[e^{j\left(\theta_{n}(z)+\theta_{H}\right)} \cdot E(z)\right]=0
$$

Solving for the field: We now look for solutions for the electric field $E(z)$ with the relaxed $\mathrm{BCs}$ of the form:

$$
E(z)=A j e^{-j \Phi(z)}
$$

In this, $A$ represent an arbitrary real constant, and $\Phi(z)$ denotes a "phase function" which is defined as the complex-valued function which is (i) analytic in the problem domain, and (ii) which complies with the BCs:

$$
\operatorname{Re}[\Phi(z)]= \begin{cases}\theta_{t}(z) & z \in \text { contact boundary } \\ \theta_{n}(z)+\theta_{H} & z \in \text { isolated boundary }\end{cases}
$$

It is elementary to show that $E(z)$ according to (6) has the required analyticity in the problem domain, and that (7) implies that the BCs (4) and (5) are fulfilled.

The phase function $\Phi(z)$ is typically easier to derive compared to the original problem, because the problem domain is not parameter-dependent, and because of the Dirichlet-type BCs (7). In fact, for some important canonical domains (for instance, the upper half plane shown in the middle of fig. 1), a closed-form solution is known for piecewise constant BCs [7, p. 77]. Hence, if one knowns the conformal map $f$ between such a canonical domain and the original problem domain, the phase function $\Phi(z)$ is in this case readily available. In order to illustrate this, we consider again the two-contact problem shown in fig. 1 (left). In this example, the orientation of the boundary is such that on contacts $\theta_{t}=0$, while on isolated parts $\theta_{n}=0$. Then (7) requires $\operatorname{Re}[\Phi]=0$ on contact boundaries, and $\operatorname{Re}[\Phi]=\theta_{H}$ on isolated boundaries. The solution in the canonical domain (we use tildes for quantities in this domain) is:

$$
\tilde{\Phi}=-j \frac{\theta_{H}}{\pi}\left[\ln \frac{w+1}{w+\frac{1}{k}}+\ln \frac{w-\frac{1}{k}}{w-1}\right]
$$

which after some small manipulations gives the electric field (6):

$$
\tilde{E}(w)=A j e^{-\frac{\theta_{H}}{\pi} \ln \left[\frac{1+w}{1+k w} \frac{1-k w}{1-w}\right]}
$$


The conformal map between the problem domain and the canonical domain is known to be [6, p. 140]

$$
z=f(w)=\int_{0}^{w} \frac{d \nu}{\sqrt{1-\nu^{2}} \sqrt{1-k^{2} \nu^{2}}} \leftrightarrow w=f^{-1}(z)=\operatorname{sn}(z, k)
$$

where $k$ is parameter which determines the geometry of the plate $(K=$ $K(k)$ and $K^{\prime}=K\left(\sqrt{1-k^{2}}\right)$ with $K$ representing the complete elliptic integral), and $\operatorname{sn}(z, k)$ is the associated Jacobi elliptic function. Hence, the electric field in the original problem domain is found in closed-form: $E(z)=\tilde{E}(\operatorname{sn}(z, k))$.

Integrating the field: With the above procedure, one has a fair hope of finding closed-form analytical solutions for the electrical field $E(z)$. According to (2), the complex potential $w_{E}$ can then be recovered by integrating $E(z)$ along a path $O P$ within the problem domain. Because in most cases no analytical expression is known for the integral, numerical integrations will come into play. There is a high flexibility in addressing the problem numerically. For instance, one can choose the integration path to optimize the accuracy, e.g. by keeping away from singularities. Moreover, by making appropriate substitutions, the integration can be carried out in other domains. For instance, with the substitution $z=f(w)$, the integration can also be carried out in the canonical domain:

$$
w_{E}(P)-w_{E}(O)=\int_{O}^{P} E(z) d z=\int_{O^{\prime}}^{P^{\prime}} \tilde{E}(w) \frac{d z}{d w} d w
$$

with $O^{\prime}=f^{-1}(O)$ and $P^{\prime}=f^{-1}(P)$. When the map is of the SchwarzChristoffel type, the derivative $\frac{d z}{d w}$ is actually the integrand of this map. E.g., for our example problem we have:

$$
w_{E}(P)-w_{E}(O)=A j \int_{O^{\prime}}^{P^{\prime}} \frac{e^{-\frac{\theta_{H}}{\pi}\left[\ln \frac{1+w}{1+k w}+\ln \frac{1-k w}{1-w}\right]}}{\sqrt{1-w^{2}} \sqrt{1-k^{2} w^{2}}} d w
$$

Note that the arbitrary real $A$ introduced in (6) also appears as a linear factor in the expressions for the complex potential, as in (12). Taking now $O$ on the lower contact, and $P$ on the upper contact, the calculated voltage difference given by $\operatorname{Re}\left[w_{E}(P)-w_{E}(O)\right]$ must be equal to the applied excitation voltage $V_{0}$. From this, the unique value of $A$ can be derived.

The (numerically calculated) complex potential $w_{E}$ provides through $V_{E}=\operatorname{Re}\left[w_{E}\right]$ information on the potential distribution within the Hall plate. Also information on currents flowing in the Hall device can be derived easily. From (3), we see that $w_{E}$ multiplied with a complexvalued scale factor gives us directly $w_{J}$. The current $I_{a b}$ flowing through an arbitrary segment $a b$ can be shown to be simply $I_{a b}=\delta$. $\operatorname{Im}\left[w_{J}(b)-w_{J}(a)\right]$, where $\delta$ represents the thickness of the sample. This can be seen from the integration of $J(z)$ in (2), and transforming the result in vector notation: $w_{J}(b)-w_{J}(a)=-\int_{a}^{b} \mathbf{J} \cdot d \mathbf{s}-j \int_{a}^{b}\left(\mathbf{e}_{z} \times \mathbf{J}\right)$. $d \mathbf{s}$. The second integral, i.e. the imaginary part, represents the flux of $\mathbf{J}$ through the $a b$ segment, which is also $I_{a b} / \delta$.

Example: In the previous section, it has been shown that voltages and currents of the Hall plate have integral representations. Starting from (8), and possibly using substitutions such as in (11), there is a fair chance that also closed-form expression for the integrand can be found. Here, we illustrate that further manipulations of the integral, e.g. using well chosen substitutions, can help for easier numeric evaluation. Due to space restrictions, we will limit ourselves to a representative example here: the calculation of the mid-length Hall voltage $V_{H}$ of a rectangular plate (fig. 1). After derivation, the following expression has been obtained:

$$
V_{H}=\left(\frac{1}{\sin \theta_{H}} \frac{\int_{0}^{+\infty} \frac{\sinh \left(\frac{\theta_{H}}{\pi} \tau\right)}{\sqrt{\cosh \tau-\gamma}} d \tau}{\int_{0}^{+\infty} \frac{\cosh \left(\frac{\theta_{H}}{\pi} \tau\right)}{\sqrt{\cosh \tau+\gamma}} d \tau}\right) V_{H \infty}
$$

In this $\gamma=1-\frac{8 k}{(1+k)^{2}}$ is a geometry-dependent parameter, and $V_{H \infty}=$ $\frac{\rho \mu_{H}}{\delta} I_{0} B_{0}$ is the Hall voltage of an infinitely long plate [1]. In deriving this result, use has been made of a change of variable from $w$ tot $t$ according to

$$
t=\ln \left[\frac{1+w}{1+k w} \frac{1-k w}{1-w}\right]
$$

This substitution has been used to transform integrals of the form (12) that exhibited endpoint singularities to a numerically more attractive alternative form. The integrals in (13) converge exponentially for $\left|\theta_{H}\right|<\frac{\pi}{2}$, and since $0 \leq \gamma<1$ there are also no singularities within the integration interval.
Numerical evaluation of (13) for various aspect ratios and values of $\theta_{H}$ has been found to be in perfect agreement with [1, fig. 4.18]. Hence, (13) provides an efficient expression for a result which is, to the authors' knowledge, only found in graphical form in literature.

Conclusion: In this letter, we have developed a new method to solve electrostatic Hall problems. We have demonstrated that when an analytic phase function $\Phi$ can be found that complies with Dirichlet-type BCs (7), then (6) provides an expression for the electric field. The (complex) potential was then derived through integration. It has been demonstrated that the integral-formulation provides much flexibility to transform the integrals involved into numerically well-conditioned forms.

J. Raman, P. Rombouts and L. Weyten, Ghent University, ELIS-CAS

E-mail: johan.raman@elis.ugent.be

\section{References}

1 R.S. Popović, "Hall effect devices", Institute of Physics, 2004

2 M.J. Moelter, J. Evans, G. Elliot, and M. Jackson, "Electric Potential in the Classical Hall Effect: An Unusual Boundary-Value Problem", American Journal of Physics, vol. 66, nr. 8, 1998, pp. 668-677.

3 G. De Mey, "Potential calculations in Hall plates," Advances in electronics and electron physics, vol. 61, 1983, pp. 1-62

4 R.F. Wick "Solution of the field problem of the Germanium gyrator," J. Appl. Phys., vol. 25, 1954, pp. 741-756

5 L.N. Trefethen, R.J. Williams, "Conformal mapping solution of Laplace's equation on a polygon with oblique derivative boundary conditions", J. Comput. Appl. Math., 1986, pp. 227-249

6 W.J. Gibbs, "Conformal transformations in electrical engineering", Chapman \& Hall, 1958

7 T.A. Driscoll, L.N. Trefethen, "Schwarz-Christoffel mapping", Cambridge University Press, 2002 